\title{
PDRs and XDRs
}

\author{
Rowin Meijerink ${ }^{1}$ \\ ${ }^{1}$ Leiden Observatory, Leiden University, \\ P.O. Box 9513, NL-2300 RA, Leiden, the Netherlands \\ email: meijerink@strw.leidenuniv.nl
}

\begin{abstract}
An overview of the important thermal and chemical processes in "photon-dominated regions" or "photo-dissociation regions" (PDRs) and "X-ray dominated regions" (XDRs) is presented. Applications of the models are shown to observations of the ultra-luminous infrared galaxy Mrk 231, and the starburst galaxy M 82.
\end{abstract}

Keywords. PDRs, XDRs, ISM, galaxies

\section{Introduction}

An overview of the theory and observations of "photon dominated Regions" or "photodissociation regions" (PDRs; far-ultraviolet; $6<E<13.6 \mathrm{eV}$ ) and "X-ray dominated regions" (XDRs; $E>1 \mathrm{keV}$ ) is presented. In PDRs and XDRs, the chemical structure and thermal balance are completely determined by the radiation field. Therefore, PDRs and XDRs are direct manifestations of the energy balance of interstellar gas, and their study allows one to determine how the interstellar medium (ISM) survives the presence of stars and AGN (Tielens \& Hollenbach 1985; Boland \& de Jong 1982; van Dishoeck \& Black 1988; Le Bourlot et al. 1993; Wolfire et al. 1993; Spaans et al. 1994; Sternberg \& Dalgarno 1995; Stoerzer et al. 1998; Spaans 1996; Bertoldi \& Draine 1996; Maloney et al. 1996; Lee et al. 1996; Kaufman et al. 1999; Le Petit et al. 2002; Meijerink \& Spaans 2005; Meijerink et al. 2007, and references therein). First, the important processes in XDRs and PDRs are discussed. UV and X-ray irradiation give rise to different gas cloud thermal/chemical structures, and therefore also show different line emission spectra. Xrays penetrate through a larger column of gas (up to $N_{\mathrm{H}} \sim 10^{24} \mathrm{~cm}^{-2}$ ) than UV photons $\left(N_{\mathrm{H}} \sim 10^{21}-10^{22} \mathrm{~cm}^{-2}\right)$, and the heating efficiency of X-rays (10-50 percent) is much larger than that of UV irradiation (less than 1 percent). X-rays also give rise to significant ionization fractions (up to $x_{\mathrm{e}} \sim 10^{-2}-10^{-1}$ ), driving an efficient ion-molecule chemistry, while UV photons are more efficient in dissociating molecules. Therefore, for comparable energy inputs, XDRs have much larger column densities of warm molecular gas than PDRs. Second, the models are applied to observations of the ISM in M 82 and Mrk 231, and the CO ladder in particular, as detected by the various Key Programs on the Herschel Space Observatory.

\section{Physical processes}

\subsection{PDRs}

The global properties of PDRs are determined by a number of physical processes: (i) heating through photo-electric emission (cf., Bakes \& Tielens 1994; Weingartner \& Draine 2001), (ii) heating by FUV pumping of $\mathrm{H}_{2}$, followed by collisional de-excitation (cf., Hollenbach \& McKee 1979), (iii) heating by cosmic rays (cf., Field et al. 1969), (iv) 


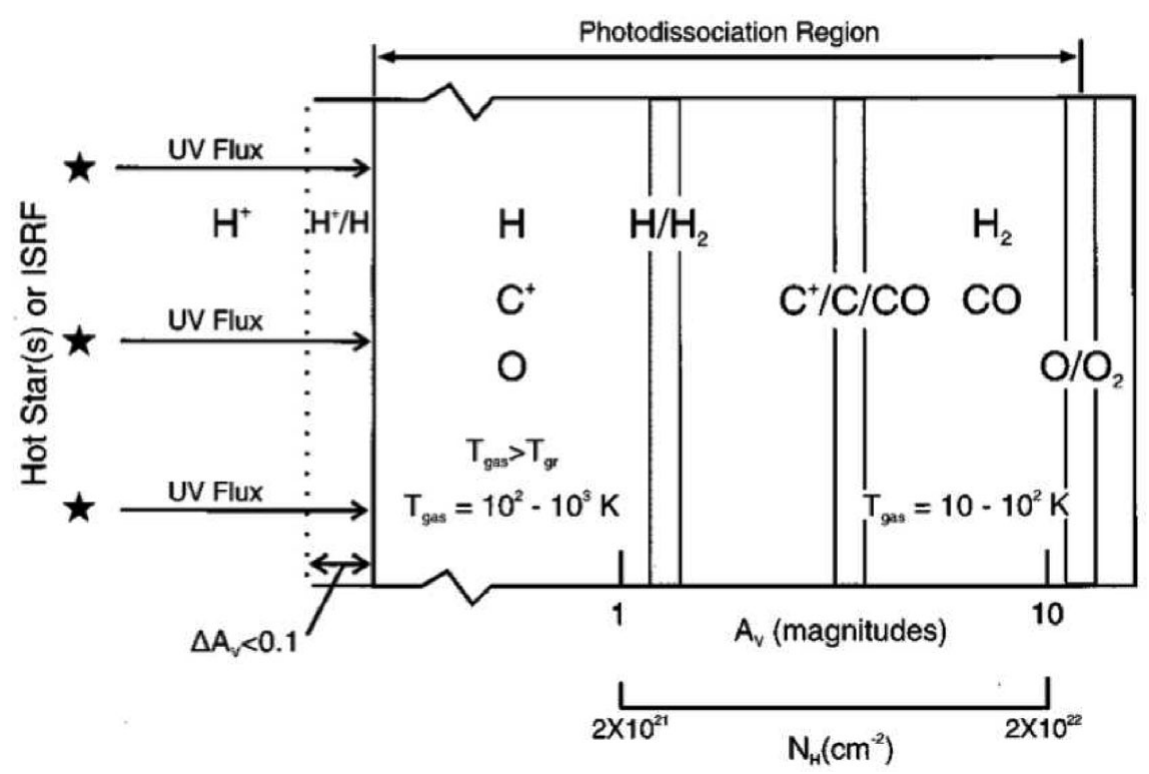

Figure 1. Schematic structure of PDR adapted from Tielens \& Hollenbach (1985).

fine-structure line cooling of [CII] $158 \mu \mathrm{m}$, [OI] $63 \mu \mathrm{m}$, [CI] $609 \mu \mathrm{m}(\mathrm{v})$ molecular line cooling by warm molecular gas containing $\mathrm{CO}, \mathrm{H}_{2}, \mathrm{H}_{2} \mathrm{O}, \mathrm{OH}$, and $\mathrm{CH}$ (cf., Neufeld et al. 1995; Spaans \& Silk 2000) (v) ion-molecule reactions driven by the ionization degree of $x_{e} \sim 10^{-4}$ maintained by the ionization of carbon in the FUV (cf., Black \& Dalgarno 1977; van Dishoeck \& Black 1986), and (vi) the ionization balance of atomic gas under the influence of photo-ionization driven by FUV photons and counteracting recombination and charge transfer reactions with metals and particularly PAHs (cf., Lepp \& Dalgarno 1988; Bakes \& Tielens 1994).

A schematic overview of a PDR is shown in Fig. 1. The thermal and chemical balance changes, as one moves into a PDR: The extinction along the line of side increases and the impinging radiation field is attenuated. There are two very fundamental chemical transitions, the $\mathrm{H} / \mathrm{H}_{2}$ and the $\mathrm{C}^{+} / \mathrm{C} / \mathrm{CO}$ transition. The first one occurs because the Lyman and Werner electronic bands, that are able to dissociate $\mathrm{H}_{2}$, become optically thick due to self-shielding. At slightly larger extinction, ionized carbon is converted into neutral carbon, as the FUV flux is attenuated by dust absorption. The transformation of neutral carbon to $\mathrm{CO}$ occurs when the FUV field is reduced by grain opacity, $\mathrm{H}_{2}$ shielding and CO self-shielding.

The region, where most hydrogen is still in atomic form and the extinction still very small ( $\sim$ few $A_{V}$ ), is usually referred to as the radical region since many carbon hydrides and their ions, e.g., $\mathrm{CH}, \mathrm{CH}^{+}, \mathrm{CN}, \mathrm{HCN}, \mathrm{HCO}^{+}$, etc., reach their peak abundance here, caused by the presence of both $\mathrm{C}^{+}$and $\mathrm{H}_{2}$ and high temperatures $\left(T \sim 10^{2}-10^{3} \mathrm{~K}\right)$. Many of the present atoms and molecules are collisionally excited at the abient densities and temperatures, and emit bright in the mid- and far-infrared, millimeterm and submillimeter.

There are four parameters that determine the global characteristerics of any PDR: (i) the strength of the impinging radiation field, in units of the Habing (1969) $G_{0}$ or Draine (1978) $I_{\mathrm{UV}}$ radiation field, (ii) the temperature and the ambient hydrogen, $n_{\mathrm{H}}=$ $n(\mathrm{H})+n\left(\mathrm{H}_{2}\right)$, set the rates of the chemical reactions and excitation of the coolants, (iii) 


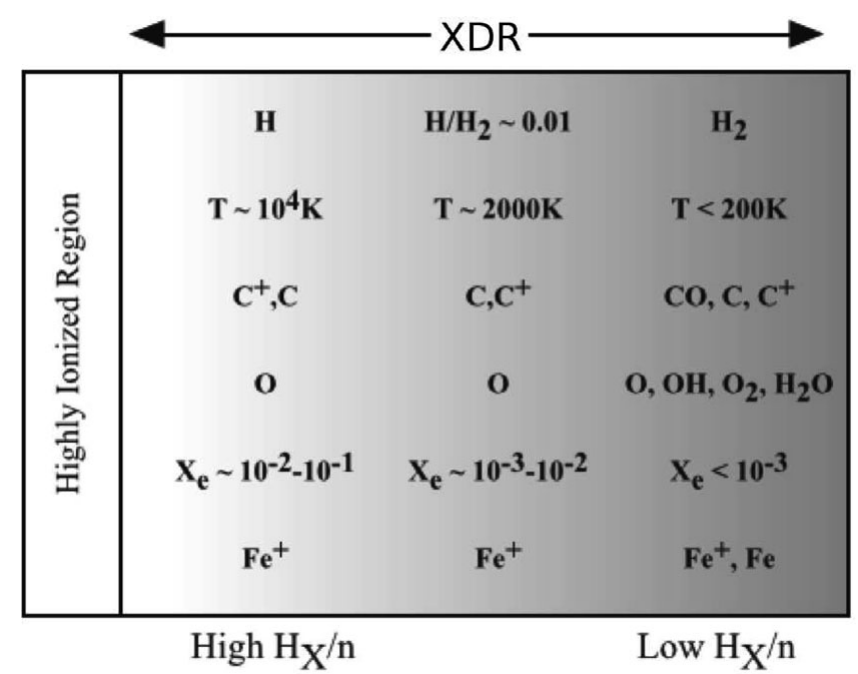

Figure 2. Schematic structure of XDR adapted from Maloney et al. (1996).

the metallicity, $Z$, in units of the solar value $Z_{\odot}$, determines the total abundances for carbon- and oxygen-bearing species, (iv) the spectral shape, characterized by the color temparature $T_{\text {eff }}$, fixes the distribution of photon flux over energy.

\section{2. $X D R s$}

The following processes are important in XDRs: (i) photo-ionization heating (i.e., Coulomb interaction of $\mathrm{keV}$ electrons with thermal electrons), which is dominant by a large factory over photo-electric heating by dust grains (Maloney et al. 1996). (ii) cooling by metastable lines of [CI] 9823, $9850 \AA$ and [OI] $6300 \AA$, fine-structure cooling by [OI] 63, $145 \mu \mathrm{m}$ and [CII] $158 \mu \mathrm{m}$, as well as Lyman $\alpha$ emission (Maloney et al. 1996; Tielens \& Hollenbach 1985; Spaans et al. 1994), (iii) molecular cooling by warm molecular gas, containing $\mathrm{CO}, \mathrm{H}_{2}, \mathrm{H}_{2} \mathrm{O}$ and $\mathrm{OH}$ as well as gas-grain cooling where warm gas is cooled a the surfaces of lower temperature dust grains (cf., Neufeld et al. 1995; Spaans \& Silk 2000) (iv) ion-molecule reactions driven by the ionization degree of $10^{-4}$ maintained by ionization of carbon (c.f., Black \& Dalgarno 1977; van Dishoeck \& Black 1986), and (v) the ionization balance of atomic gas under the influence of photo-ionization reactions driven by X-ray photons and charge transfer (c.f., Lepp \& Dalgarno 1988; Maloney et al. 1996).

A schematic overview of an XDR is shown in Fig. 2. The global structure of the XDR is characterized by the density $n_{\mathrm{H}}$ and the energy deposition rate $H_{\mathrm{X}}$ per hydrogen atom. The heating in XDRs is driven by photo-ionization, and the heating efficiency is much larger in XDRs than in PDRs, where this is between 0.3 and 1.0 percent. The gas in XDRs is exposed to both X-ray and FUV, that is internally created by excitation of the Lyman-Werner and Lyman $\alpha$ bands. Attenuation of X-rays is done by atomic electronic absorptions. The lowest energy photons are attenuated most strongly. 


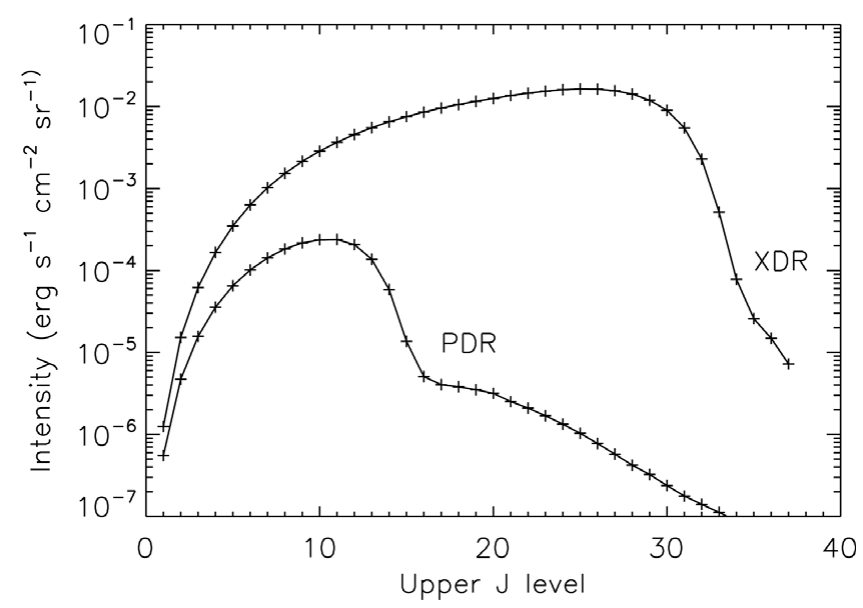

Figure 3. Comparison of PDR and XDR CO ladders (Meijerink 2006; Spaans \& Meijerink 2008).

\section{Differences and consequences for diagnostics}

There are four very important differences between PDRs and XDRs: (i) X-rays penetrate much larger column densities than UV photons, (ii) the gas heating efficiencies in XDRs range from 10 to 50 percent, which is much larger than the $<1 \%$ in PDRs, (iii) dust heating is much more efficient in PDRs than in XDRs, and (iv) high ionization levels in XDRs drive an ion-molecule chemistry over a large column density.

As a result, XDRs produce larger column densities of warmer gas. Thus, identical incident energy densities give very different emergent continuum and line spectra. One example is given in Fig. 3 (Meijerink 2006; Spaans \& Meijerink 2008), where the CO line spectral energy distribution is given for a model with density $n=10^{5} \mathrm{~cm}^{-3}$ and $G_{0}=10^{5}\left(F_{X}=160 \mathrm{erg} \mathrm{cm}^{-2} \mathrm{~s}^{-1}\right)$. The CO ladder for the PDR model turns over at the $J=10-9$ transition, while CO fluxes of the XDR continue rising up to $J=26-25$, for this particular model. It is therefore expected, that high- $J$ CO lines are excellent XDR tracers, when a good sampling of the CO ladder is obtained.

\section{Application of PDR and XDR models}

The predictions derived from the PDR and XDR models, were in part the motivation for the Herschel Open Time Key Program HerCULES (PI: P.P. van der Werf), which uniformly and statistically measures the neutral gas cooling lines in a flux limited sample of 29 (U)LIRGs, selected from the Revised IRAS Bright Galaxy Sample (Sanders et al. 2003). The sample consists of 6 ULIRGs $\left(S_{60}>12.19 \mathrm{Jy}\right)$ and 23 LIRGs $\left(S_{60}>16.8\right.$ Jy). Full high-resolution SPIRE FTS scan are made between wavelengths $\lambda \sim 200$ to $670 \mu \mathrm{m}$, covering $\mathrm{CO}(4-3)$ to $\mathrm{CO}(13-12)$, [CI], a number of $\mathrm{H}_{2} \mathrm{O}$ lines, and several other bright lines. Also, PACS line scans of the [CII] $158 \mu \mathrm{m}$ and [OI] 63,145 $\mu \mathrm{m}$ lines are obtained.

\subsection{Mrk 231}

One source in this sample is Mrk 231, the most luminous galaxy in the Revised IRAS Bright Galaxy Sample (Sanders et al. 2003). It has a redshift $z=0.042170$ and a 


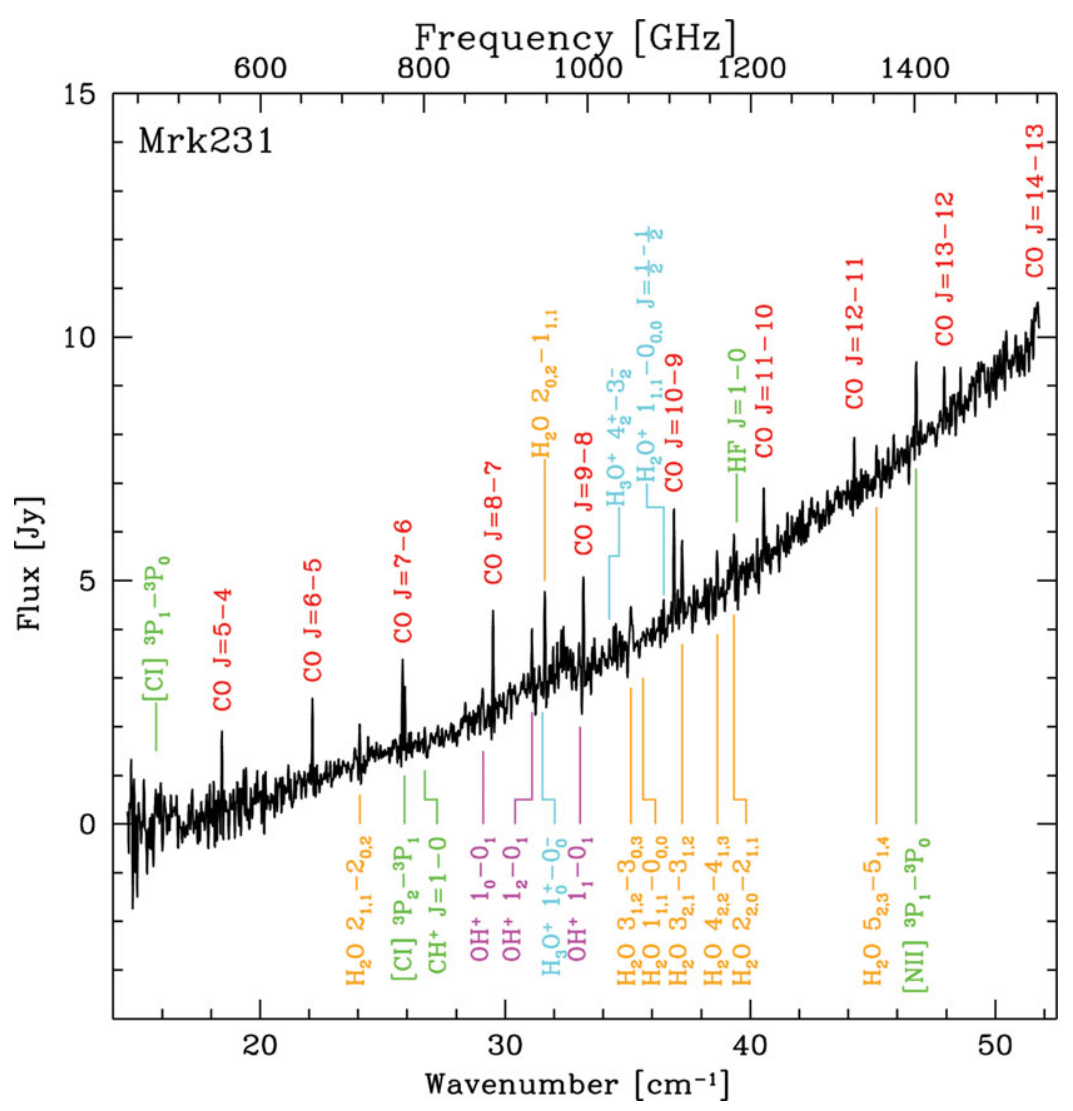

Figure 4. SPIRE FTS Spectrum Mrk 231 (van der Werf et al. 2010).

luminosity distance $D_{L}=192 \mathrm{Mpc}, L_{\mathrm{IR}}(8-1000 \mu \mathrm{m})=4.0 \times 10^{12} \mathrm{~L}_{\odot}$. Mrk 231 contains a luminous, optically visible AGN, classified as s Seyfert 1 or a broad absorption line QSO (Boksenberg et al. 1977). A highly absorbed power-law X-ray spectrum was observed by Braito et al. (2004) with $L_{\mathrm{X}}=6_{-0.3}^{+0.6} \times 10^{43} \mathrm{erg} \mathrm{s}^{-1}$ between 2 and $10 \mathrm{keV}$, so it is an excellent candidate to observe XDR signatures in the line emission. However, Mrk 231 also contains a kpc size disk harbouring intense star formation (Taylor et al. 1999), so a PDR contribution is also expected.

The obtained SPIRE FTS spectrum is shown in Fig. 4 (van der Werf et al. 2010). It contains at least the following lines: $10 \times \mathrm{CO}(5-4$ to $14-13), 2 \times[\mathrm{CI}],[\mathrm{NII}], 8 \times \mathrm{H}_{2} \mathrm{O}, 3 \times$ $\mathrm{OH}^{+}, \mathrm{H}_{2} \mathrm{O}^{+}, 2 \times \mathrm{H}_{3} \mathrm{O}^{+}, \mathrm{CH}^{+}$, and HF. There are still an number of unidentified lines at this point. Very surprising are the bright emission lines from $\mathrm{OH}^{+}$and $\mathrm{H}_{2} \mathrm{O}^{+}$, which are highly reactive, transient ionic species. Furthermore, the water lines have strenghts of 20-50 percent of the CO lines. This is not observed for the Orion Bar or the starburst galaxy M 82, where the strength of the water lines relative to $\mathrm{CO}$ is at least an order of magnitude lower.

A fit to the CO lines is shown in Fig. 5: a diffuse PDR component $\left(n=10^{3.5} \mathrm{~cm}^{-3}\right.$, $\left.G_{0}=10^{2}\right)$ and a high density $\left(n=10^{5}, G_{0}=10^{3.5}\right)$ embedded PDR component are needed to fit the lower CO transition $(J<10-9)$, while for the higher- $J$ CO lines an XDR component is fitted. This last component has a density $n=10^{4.2} \mathrm{~cm}^{-3}$ and incident 


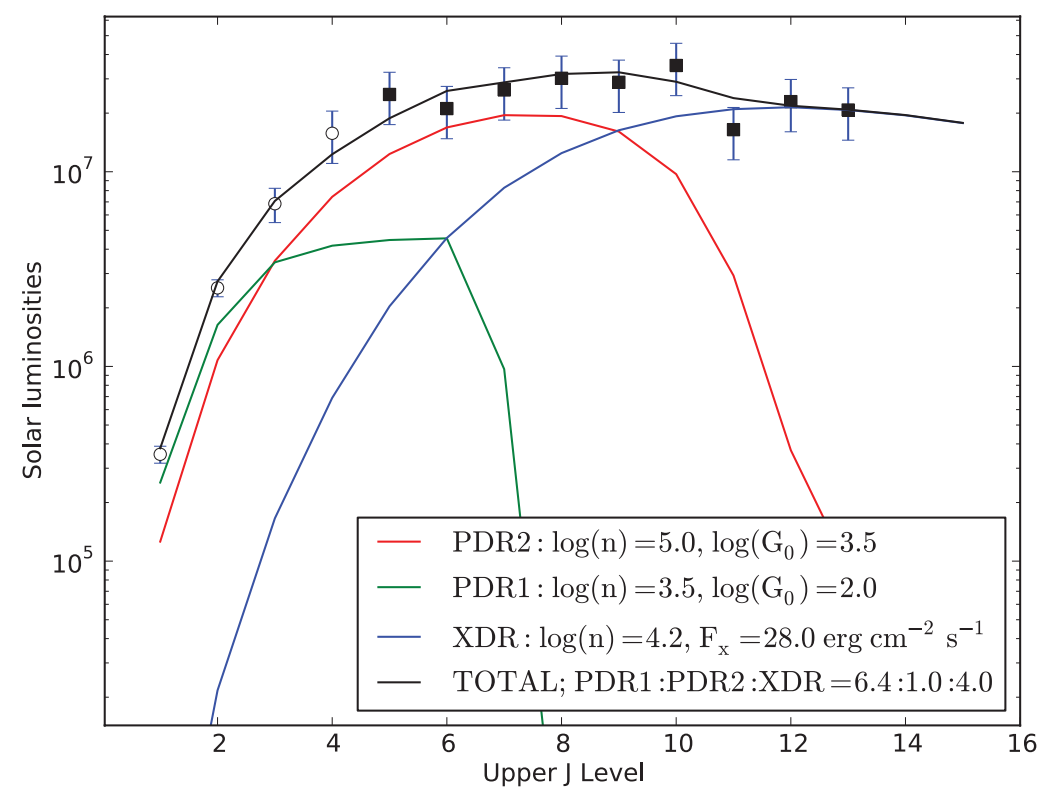

Figure 5. PDR/XDR fit to the CO ladder of Mrk 231 (van der Werf et al. 2010).

flux $F_{x}=28 \mathrm{erg} \mathrm{s}^{-1} \mathrm{~cm}^{-2}$, a flux that can be produced out to a distance of $160 \mathrm{pc}$, with the observed AGN. The surface area ratios between the different component is PDR1: PDR2: XDR = 5.4:1.0:4.0.

It is also possible to fit a very dense, high illumination PDR to the very high- $J$ CO lines, with $n=10^{6.5} \mathrm{~cm}^{-3}$ and $G_{0}=10^{5}$. The ratio between the medium and high excitation component is 1.0:0.03. Here the smal surface are for the high excitation PDR indicates a number of small high density clumps in a very strong UV field. Since the radiating surface of the high excitation PDR is about $30 \times$ smaller than that of the medium excitation $\mathrm{PDR}$, but its density about $30 \times$ larger, the $\mathrm{H}_{2}$ masses in these two components must be comparable. In this scenario about half of the mass would be contained in $0.7 \%$ of the volume, and the dust in this component would be heated to $T_{\text {dust }}=170 \mathrm{~K}$, which is simply not observed for Mrk 231. Therefore, the possible presence of an extreme PDR component is not very likely.

\section{2. $M 82$}

It is very illustrative to compare the CO observations of Mrk 231 to those obtained for M 82 with Herschel HIFI (Loenen et al. 2010) and SPIRE (Panuzzo et al. 2010), and combined with ground based data (Ward et al. 2003). The two CO SLEDs, for ${ }^{12} \mathrm{CO}$ and ${ }^{13} \mathrm{CO}$ are shown in Fig. 6 . The observations can be best reproduced with a combination of one low and two high-density components. The low-density component, which dominates in the lowest CO lines $(J \leqslant 3)$, is found to have a density of $n=10^{3.5} \mathrm{~cm}^{3}$ and a UV flux of $G_{0}=10^{2}$. This component represents the extended molecular ISM. The mid- $J$ CO lines $(4<J<7)$ are mostly produced by clouds that have a density of $n=10^{5} \mathrm{~cm}^{3}$ and a radiation field of $G_{0}=10^{2.75}$. At transitions higher than 7 (and ${ }^{13} \mathrm{CO} J>5$ ), the line emission comes from clouds with even higher densities $\left(n=10^{6} \mathrm{~cm}^{3}\right)$ and radiation fields $\left(G_{0}=10^{3.25}\right)$. These two models represent the dense, star-forming molecular clouds. 


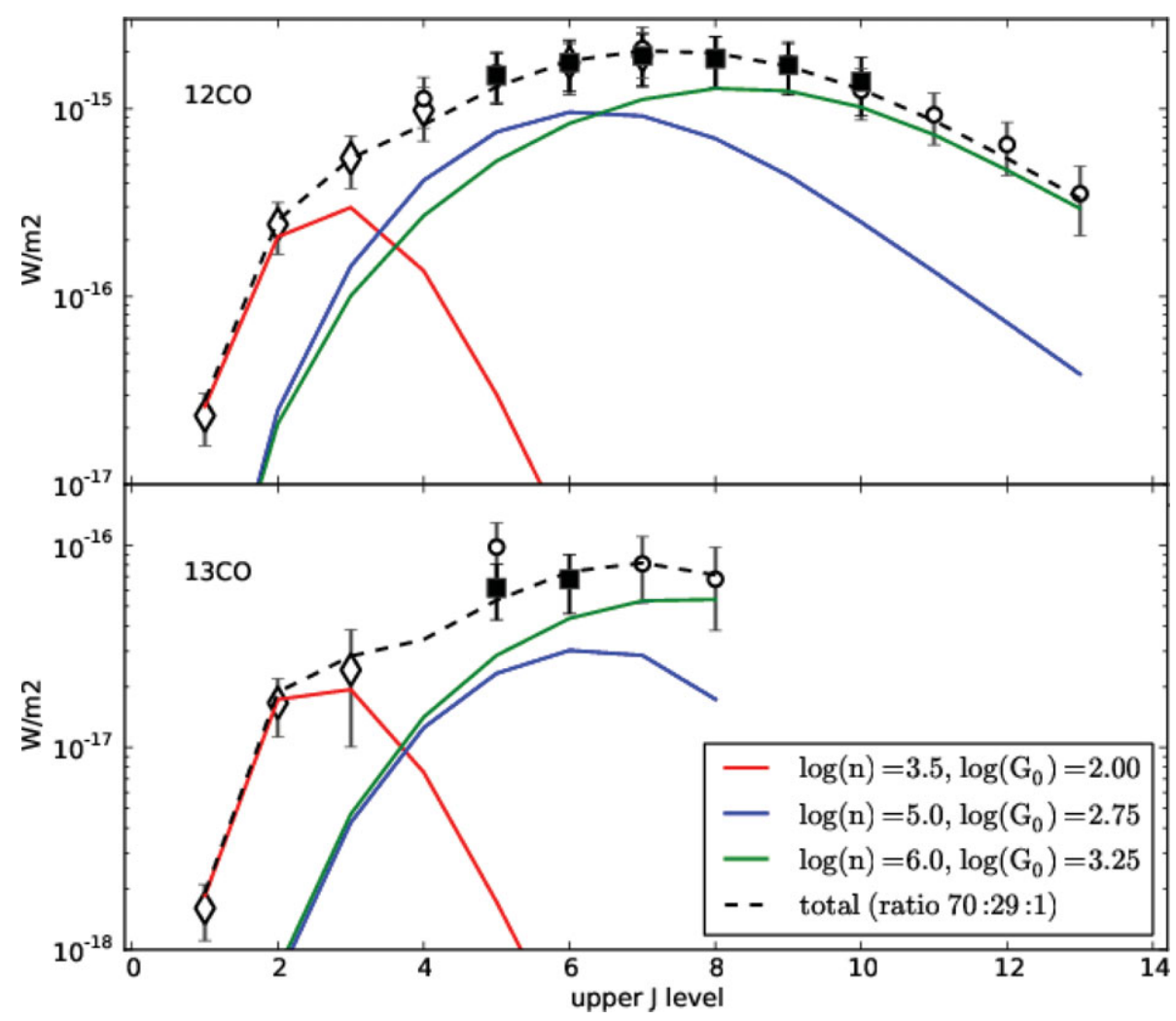

Figure 6. PDR fit to the ${ }^{12} \mathrm{CO}$ and ${ }^{13} \mathrm{CO}$ ladders of Mrk 82 (Loenen et al. 2010).

\subsection{Other diagnostics and caveats}

A major issue in the study of the interstellar medium in galaxies is that a single pointing usually contains a mix of components that is affected by different physical processes. It may contain (i) quiescent molecular and atomic gas (ii) star forming molecular gas (PDRs), (iii) AGN (X-ray) excited gas (XDRs), (iv) cosmic ray heated gas, (v) shocks (vi) mechanically (dissipation of turbulence) heated gas, and (vi) warm, very obscured gas (hot cores). As we saw for Mrk 231, it is possible to reproduce the high- $J$ CO lines with an XDR component or a very extreme PDR component. Also, when a normal PDR component receives additional heating from, e.g., dissipating turbulence, it is possible to produce a flat CO ladder for Mrk 231 for the very high- $J$ CO lines.

Additional information is given by other tracers. Bright $\mathrm{OH}^{+}$and $\mathrm{H}_{2} \mathrm{O}^{+}$lines have been detected in Mrk 231. The estimated lower limits $\mathrm{OH}^{+}$and $\mathrm{H}_{2} \mathrm{O}^{+}$abundances relative to $\mathrm{H}_{2}$ are of order $2 \times 10^{-10}$ in the central $160 \mathrm{pc}$. Such abundances require an efficient and penetrative source of ionization in the molecular gas, since the production of $\mathrm{OH}^{+}$is mainly driven by $\mathrm{H}^{+}+\mathrm{O} \rightarrow \mathrm{O}^{+}+\mathrm{H}$ followed by $\mathrm{O}^{+}+\mathrm{H}_{2} \rightarrow \mathrm{OH}^{+}+\mathrm{H}$ and $\mathrm{H}^{+}+\mathrm{OH} \rightarrow$ $\mathrm{OH}^{+}+\mathrm{H}$. The key species $\mathrm{H}^{+}$is 2 to 3 orders of magnitude more abundant in the XDR model than in the high excitation $\mathrm{PDR}$, and the $\mathrm{OH}^{+}$abundance is larger by a comparable amount, see Fig. 7. A similar argument can be made for $\mathrm{H}_{2} \mathrm{O}^{+}$, which is formed by $\mathrm{OH}^{+}+\mathrm{H}_{2} \rightarrow \mathrm{H}_{2} \mathrm{O}^{+}+\mathrm{H}$. The extraordinary luminosity of the $\mathrm{OH}^{+}$and $\mathrm{H}_{2} \mathrm{O}^{+}$ (and to a lesser extent $\mathrm{CH}^{+}$) lines in Mrk 231 is underlined by a comparison with the 

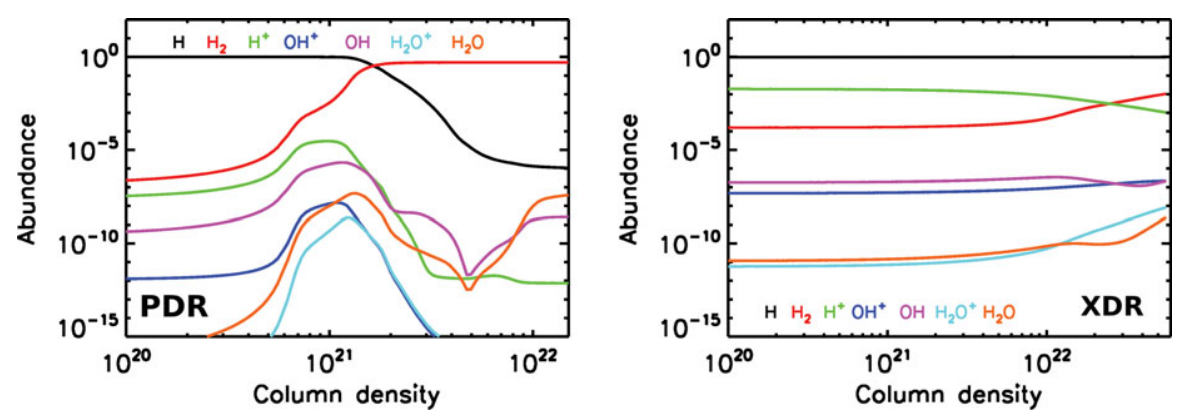

Figure 7. An extreme PDR and XDR used to fit the high- $J$ CO lines (based on Meijerink et al. 2007). Note the overall much higher abundances of $\mathrm{H}^{+}, \mathrm{OH}^{+}$, and $\mathrm{H}_{2} \mathrm{O}^{+}$in the $\mathrm{XDR}$.

SPIRE spectrum of M82 and also the Orion bar PDR (Habart et al. 2010), which show no trace of $\mathrm{OH}^{+}$or $\mathrm{H}_{2} \mathrm{O}^{+}$, and only weak $\mathrm{CH}^{+}$emission in the Orion Bar, while in Mrk 231 the lines are only a factor 2 to 3 fainter than the CO lines.

Enhanced cosmic ray fluxes in a starburst environment will increase the degree of ionization and hence the production of $\mathrm{OH}^{+}$and $\mathrm{H}_{2} \mathrm{O}^{+}$in a PDR (Meijerink et al. 2011), but cosmic ray fluxes have to be 2 to 3 orders of magnitude higher to obtain similar abundances as seen in the XDR. Also, they do not elevate the temperatures to the level required to produce the highest CO lines (Meijerink et al. 2006). It is thus the combination of strong high- $J \mathrm{CO}$ lines and high $\mathrm{OH}^{+}$and $\mathrm{H}_{2} \mathrm{O}^{+}$abundances that reveals X-ray driven excitation and chemistry in Mrk 231.

\section{Summary \& Conclusions}

The Herschel Space Observatory opened a very new window in the study of the ISM in nearby galaxies. The SPIRE FTS instrument, covering the wavelength range between $\lambda=200-670 \mu \mathrm{m}$, contains a whealth of diagnostic lines, such as the CO $J=5-4$ to $13-12$, water lines with excitation temperatures up to $T \sim 500 \mathrm{~K}$, and transient species $\mathrm{OH}^{+}$and $\mathrm{H}_{2} \mathrm{O}^{+}$.

This allowed us to study the excitation conditions of the gas in Mrk 231, and we found that both star formation as well as black hole accretion have to be considered simultaneously in order to model the obtained CO ladder. However, the PDR/XDR fit is not unique when considering only the $\mathrm{CO}$ lines, and information on dust temperature and other species has to be considered as well.

The spectra obtained in the HerCULES Open Time Key Program will be unique templates for the study of the ISM in high-redshift galaxies with ALMA. This will allow us, in combination with the PDR/XDR models, to characterize the star forming gas in the early universe.

\section{References}

Bakes, E. L. O. \& Tielens, A. G. G. M. 1994, ApJ, 427, 822

Bertoldi, F. \& Draine, B. T. 1996, ApJ, 458, 222

Black, J. H. \& Dalgarno, A. 1977, ApJS, 34, 405

Boksenberg, A., Carswell, R. F., Allen, D. A., Fosbury, R. A. E., Penston, M. V., \& Sargent, W. L. W. 1977, MNRAS, 178, 451

Boland, W. \& de Jong, T. 1982, ApJ, 261, 110 
Braito, V., et al. 2004, A\& $A, 420,79$

Draine, B. T. 1978, ApJS, 36, 595

Field, G. B., Goldsmith, D. W., \& Habing, H. J. 1969, ApJL, 155, L149

Fischer, J., et al. 2010, A\&AA, 518, L41

Habart, E., et al. 2010, A\&A, 518, L116

Habing, H. J. 1969, Bull. Astr. Inst. Netherlands, 20, 177

Hollenbach, D. \& McKee, C. F. 1979, ApJS, 41, 555

Kaufman, M. J., Wolfire, M. G., Hollenbach, D. J., \& Luhman, M. L. 1999, ApJ, 527, 795

Le Bourlot, J., Pineau Des Forets, G., Roueff, E., \& Flower, D. R. 1993, A\&\&A, 267, 233

Lee, H.-H., Herbst, E., Pineau des Forets, G., Roueff, E., \& Le Bourlot, J. 1996, A\&A, 311, 690

Le Petit, F., Roueff, E., \& Le Bourlot, J. 2002, A\&A, 390, 369

Lepp, S. \& Dalgarno, A. 1988, ApJ, 324, 553

Loenen, A. F., et al. 2010, A\&\&A, 521, L2

Maloney, P. R., Hollenbach, D. J., \& Tielens, A. G. G. M. 1996, ApJ, 466, 561

Meijerink, R. 2006, Ph.D. Thesis, Leiden Observatory

Meijerink, R. \& Spaans, M. 2005, A\&A, 436, 397

Meijerink, R., Spaans, M., \& Israel, F. P. 2006, ApJL, 650, L103

Meijerink, R., Spaans, M., \& Israel, F. P. 2007, A $\mathscr{J} A$, 461, 793

Meijerink, R., Spaans, M., Loenen, A. F., \& van der Werf, P. P. 2011, A\& A, 525, A119

Neufeld, D. A., Lepp, S., \& Melnick, G. J. 1995, ApJS, 100, 132

Panuzzo, P., et al. 2010, A\& A, 518, L37

Sanders, D. B., Mazzarella, J. M., Kim, D.-C., Surace, J. A., \& Soifer, B. T. 2003, AJ, 126, 1607

Spaans, M. 1996, A\& A, 307, 271

Spaans, M., Tielens, A. G. G. M., van Dishoeck, E. F., \& Bakes, E. L. O. 1994, ApJ, 437, 270

Spaans, M. \& Meijerink, R. 2008, ApJL, 678, L5

Spaans, M. \& Silk, J. 2000, ApJ, 538, 115

Sternberg, A. \& Dalgarno, A. 1995, ApJS, 99, 565

Stoerzer, H. \& Hollenbach, D. 1998, ApJ, 495, 853

Taylor, G. B., Silver, C. S., Ulvestad, J. S., \& Carilli, C. L. 1999, ApJ, 519, 185

Tielens, A. G. G. M. \& Hollenbach, D. 1985, ApJ, 291, 722

van der Werf, P. P., et al. 2010, A\&A, 518, L42

van Dishoeck, E. F. \& Black, J. H. 1986, ApJS, 62, 109

van Dishoeck, E. F. \& Black, J. H. 1988, ApJ, 334, 771

Ward, J. S., Zmuidzinas, J., Harris, A. I., \& Isaak, K. G. 2003, ApJ, 587, 171

Weingartner, J. C. \& Draine, B. T. 2001, ApJS, 134, 263

Wolfire, M. G., Hollenbach, D., \& Tielens, A. G. G. M. 1993, ApJ, 402, 195

\section{Discussion}

D. FiELD: Could you use $\mathrm{OH}$ megamasers maps and assounded structural knowledge to create more detailed models

R. MeiJerink: That is a very useful suggestion. I will take a look at the available observations to see whether it will better constrain the geometric, that is currently being constructed.

S. Muller: Did you see the broad wings due to the molecular outflow in your spectra of Mrk 231

R. MEIJERINK: No it is not observed in the SPIRE spectrum, as the spectral resolution is only of order $\sim 1500 \mathrm{~km} / \mathrm{s}$. They are however observed in the $\mathrm{OH}$ lines with PACS (Fischer et al. 2010). 
D. SEmEnov: How sensitive are your results of the XDR chemical modeling to the shape $\&$ hardness of the X-ray spectrum.

R. Meijerink: We are using a power law spectrum. Making the spectrum shallower, will resul in a more extended XDR, and a temperature profile with a less strong gradient.

D. LIs: Mrk 231 is the only source that I know where HF is seen in emission. Is HF included in your models and, if so, can you reproduce the observed line intensity

R. MeiJerink: It is currently not in the code, but it can be easily added. I have thus not tried to reproduce the HF line this far. 